\title{
Microscopic bladder neck involvement by prostate carcinoma in radical prostatectomy specimens is not a significant independent prognostic factor
}

\author{
Ming Zhou ${ }^{1,2}$, Alwyn M Reuther ${ }^{2}$, Howard S Levin ${ }^{1}$, Sara M Falzarano ${ }^{1}$, Emmanuel \\ Kodjoe $^{1}$, Jonathan Myles ${ }^{1}$, Eric Klein ${ }^{2}$ and Cristina Magi-Galluzzi ${ }^{1,2}$ \\ ${ }^{1}$ Department of Anatomic Pathology, Cleveland Clinic, Cleveland, OH, USA and ${ }^{2}$ Glickman Urological and \\ Kidney Institute, Cleveland Clinic, Cleveland, OH, USA
}

\begin{abstract}
The independent prognostic importance of microscopic bladder neck involvement by prostate cancer in radical prostatectomy is questionable. We studied a cohort of 1845 patients to determine the significance of microscopic bladder neck involvement. Bladder neck involvement was defined as prostate cancer present within the coned bladder neck. We further categorized the cases as 'true bladder neck involvement' and 'false bladder neck involvement.' True bladder neck involvement required prostate cancer within thick smooth muscle bundles without intermixed benign prostatic glands. False bladder neck involvement was characterized by prostate cancer intermixed with benign prostatic glands. Bladder neck involvement was analyzed in relation to preoperative serum prostate-specific antigen (PSA) level, extraprostatic extension, seminal vesicle involvement, positive surgical margin, lymph node involvement, radical prostatectomy Gleason score, and tumor volume. Of the 90 patients $(4.9 \%)$ with microscopic bladder neck involvement, 63 were further classified as true bladder neck involvement and 27 as false bladder neck involvement. In univariate model, both types of bladder neck involvement $(P<0.001)$, true $(P<0.001)$, and false $(P=0.040)$, were significantly associated with increased PSA-recurrence risk compared to bladder neck negative cases. In multivariate model the PSA-recurrence relative risk associated with bladder neck involvement (true or false) was not a significant independent prognostic factor. Extraprostatic extension, seminal vesicle involvement, positive surgical margin, lymph node involvement, PSA, and Gleason score were significant independent predictors of PSA recurrence. The time to biochemical recurrence in patients with bladder neck involvement was similar to that of PT2 with positive surgical margin or $\mathrm{pT} 3 \mathrm{a}$ with negative surgical margin patients (Kaplan-Meier curves). Bladder neck involvement was associated with other adverse pathologic features, but was not an independent predictor of PSA recurrence. In view of the previous and current data, the staging system for bladder neck involvement should be revised and patients may be best categorized as having pT3a disease.
\end{abstract}

Modern Pathology (2009) 22, 385-392; doi:10.1038/modpathol.2008.190; published online 28 November 2008

Keywords: prostate; carcinoma; bladder neck; stage; pT3; prognostic factor

Radical prostatectomy is one of the most effective treatment modality for prostate cancer. The advantage of radical prostatectomy over other treatment options (ie brachytherapy, external beam radiation, cryoablation) is related to the possibility of obtaining a complete pathological characterization of the removed prostate. Many pathologic parameters

Correspondence: Dr C Magi-Galluzzi, MD, PhD, Department of Anatomic Pathology, Cleveland Clinic, L25, 9500 Euclid Avenue, Cleveland, OH 44195, USA.

E-mail: magic@ccf.org

Received 5 June 2008; revised 13 October 2008; accepted 19

October 2008; published online 28 November 2008 identifiable in the specimen, and characteristic of each prostate cancer removed, are of important value in predicting the likelihood of local and distant disease recurrence, so that the chance of cure can be accurately estimated and the need for additional treatment determined.

The TNM classification system established by the American Joint Committee on Cancer and International Union against Cancer (AJCC/UICC) has been widely adopted for the clinical and pathological classification of prostate cancer. ${ }^{1,2}$ Accurate pathological $\mathrm{T}$ staging should reflect the natural history of cancer and be able to stratify patients into different prognostic groups. Prostate cancer with bladder 
neck involvement was initially considered as advanced disease, similar to external sphincter and/or rectal involvement and categorized as pT4 disease. ${ }^{3}$

Considering that bladder neck-sparing techniques have been implemented in radical prostatectomy to improve postoperative urinary continence and that clinical stage pT4 prostate cancer is rarely treated surgically, this outdated staging system was based upon the urologist finding gross invasion of the bladder neck or external sphincter. Thus, the pathological definition of stage pT4 disease has referred recently almost exclusively to the incidental microscopic finding of tumor cells among smooth muscle bundles of the bladder neck.

However, several recent studies have raised the question as to whether bladder neck invasion should be considered as pT3a or pT4a disease, as these studies showed that the prognosis of patients with bladder neck involvement was no worse than those with pT3 disease. ${ }^{4-8}$ The College of American Pathologists practice protocol on prostate specifically states that 'microscopic involvement of bladder neck muscle fibers in radical prostatectomy specimens should not be equated with a pT4 designation.' pT4 generally requires 'gross involvement of the bladder neck. ${ }^{9}$

It is also controversial as to whether microscopic bladder neck invasion provides independent prognostic significance and predicts disease progression after radical prostatectomy. Several studies have shown that microscopic bladder neck invasion is not an independent predictor of progression after radical prostatectomy, and the prognosis of patients with bladder neck invasion is more dependent on other pathologic features such as extraprostatic extension, seminal vesicle invasion, positive lymph nodes, and positive surgical margins. ${ }^{5,7}$ In contrast, Cheng et $a l^{10}$ found that bladder neck invasion is still a significant predictor of disease progression. This discrepancy is in part because of inconsistency in the pathological definition of bladder neck invasion: some authors discuss bladder neck involvement reporting what others would describe as margin status at the bladder neck..$^{5,11-14}$

To elucidate the significance of microscopic bladder neck involvement in radical prostatectomy specimens and to determine the importance of a uniform definition of bladder neck involvement, we studied a large cohort of patients with prostate cancer who underwent radical prostatectomy as monotherapy at a single institution.

\section{Materials and methods}

After excluding from analysis patients treated with neoadjuvant and adjuvant therapy, our study group included 1845 consecutive patients treated with radical prostatectomy at our institution between 1996 and 2005.
The radical prostatectomy specimens were received fresh from the operating room, measured, weighed, inked in two colors (blue on the right side and yellow on the left), and fixed overnight in $10 \%$ buffered formalin. The apex of the prostate was shaved perpendicular to the prostatic urethra. The bladder neck margin was coned from the radical prostatectomy and sectioned perpendicularly. The remaining prostate was completely sectioned at $3 \mathrm{~mm}$ intervals in a plane perpendicular to the urethral axis. The prostate slices were then subdivided in quadrants and labeled to allow for reconstruction as whole-mount sections. Sections of the entire posterior peripheral zone and selected areas of the transition and anterior zone were submitted for microscopic evaluation. Blocks were embedded in paraffin and a $4-5 \mu \mathrm{m}$ section from each block was stained with hematoxylin and eosin.

Pathologic features, such as radical prostatectomy tumor Gleason score, extraprostatic extension, seminal vesicle invasion, positive surgical margin, positive lymph nodes, tumor volume, and bladder neck involvement with or without concomitant positive surgical margins, were recorded for each specimen by urologic pathologists and collected prospectively in an IRB approved database. The Gleason score was obtained by summing the primary and secondary Gleason pattern grade based on microscopic evaluation of the prostatectomy specimen. Extraprostatic extension was defined as the spread of tumor beyond the fibromuscular capsule into the periprostatic adipose tissue. Seminal vesicle invasion was diagnosed if tumor invaded the seminal vesicle muscular wall. Surgical margins were considered positive when tumor cells/glands where touching the inked surface of the prostate. Tumor volume was categorized as low $(<0.5 \mathrm{ml})$, medium $(0.5-2 \mathrm{ml})$ or extensive $(>2 \mathrm{ml})$ based on the sum of the areas of the two largest cancer foci. This method of visual estimation has been previously described and shown to have excellent correlation with actual tumor volumes as measured by computer-assisted image analysis. ${ }^{15}$ For the patients categorized as positive for microscopic bladder neck invasion, the radical prostatectomy specimens were reviewed by one of the urologic pathologists (CMG).

Bladder neck involvement was defined as the presence of neoplastic cells within the coned bladder neck. These cases were rereviewed for this particular study and subcategorized in 'true bladder neck' and 'false bladder neck' involvement. The definition of true bladder neck involvement required the presence of neoplastic cells within thick smooth muscle bundles of the coned bladder neck in the absence of intermixed benign prostatic glandular tissue on the corresponding slide. Cases with neoplastic cells within smooth muscle bundles intermixed with benign prostatic glands represented surgical incision into the prostate gland close to the 
bladder neck; therefore those cases were regarded as false bladder neck invasion.

Bladder neck involvement was analyzed in relation to serum preoperative prostate-specific antigen (PSA) level, extraprostatic extension, seminal vesicle invasion, positive surgical margin, positive lymph nodes, Gleason score, and tumor volume.

PSA recurrence (biochemical failure) was defined as a serum PSA $\geq 0.3 \mathrm{ng} / \mathrm{ml}$ after radical prostatectomy and rising, or initiation of salvage therapy. This definition was based on the observation that some patients' PSA fluctuated between 0.1 and $0.3 \mathrm{ng} / \mathrm{ml}$ for some time, but once the PSA reached $0.4 \mathrm{ng} / \mathrm{ml}$, it never decreased. ${ }^{16}$ Time to progression in months was defined as the first PSA $\geq 0.3 \mathrm{ng} / \mathrm{ml}$ or date of initiation of salvage therapy, subtracted by the date of surgery.

\section{Statistical Analysis}

Bladder neck involvement was analyzed in relation to PSA, extraprostatic extension, seminal vesicle invasion, positive lymph nodes, positive surgical margin, radical prostatectomy Gleason score, and tumor volume. Fisher's exact or $\chi^{2}$-tests were used to test for a difference between bladder neck and categorical variables. Unpaired $t$-test was used to assess the difference between bladder neck and continuous variables. Progression-free probability was estimated using the Kaplan-Meier method and log-rank $P$-values.

To better categorize the weight of bladder neck involvement relative to other pathological parameters, we compared time to PSA recurrence using Cox proportional hazards models in different pathological groups, including (1) organ-confined disease, negative surgical margin (pT2); (2) bladder neck involvement, positive surgical margin, negative seminal vesicles invasion; (3) bladder neck involvement, negative surgical margin, negative seminal vesicles invasion; (4) pT2 with positive surgical margin; (5) pT3a with negative surgical margin; (6) pT3a with positive surgical margin; (7) pT3b. Statistical significance for all analyses were achieved at $P<0.05$. Calculations were performed by StatView software version 5.0 (SAS Institute Inc., Cary, NC, USA).

\section{Results}

A total of 1845 men underwent radical prostatectomy as monotherapy for prostate cancer at our institution between 1996 and 2005. Microscopic bladder neck involvement was present in 90 (4.9\%) patients included in our study. The radical prostatectomy specimens of patients with bladder neck invasion were subcategorized in true bladder neck (63/90, 70\%; Figure 1) and false bladder neck involvement (27/90, 30\%; Figure 2).

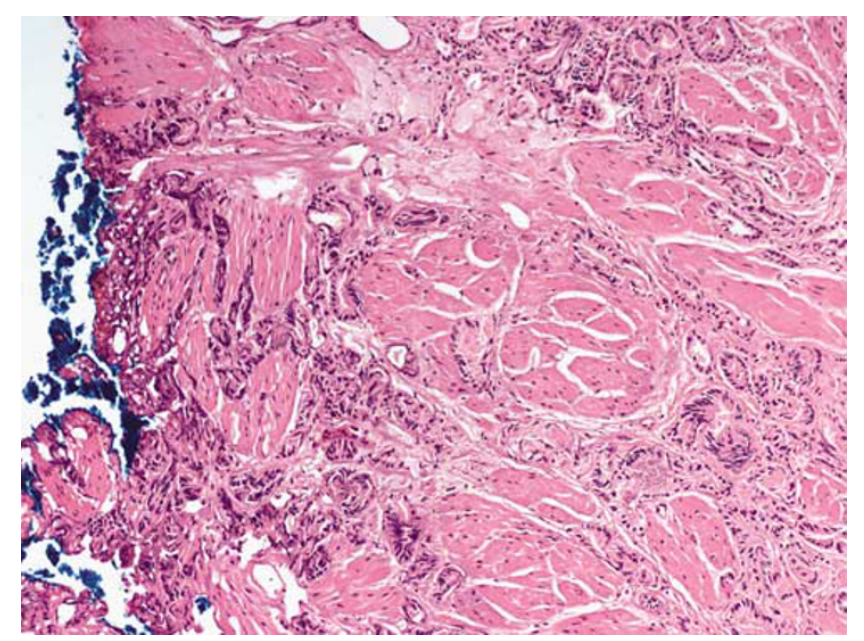

Figure 1 True bladder neck involvement showing prostate cancer within thick smooth muscle bundles of the bladder neck in absence of intermixed benign prostatic glandular tissue.

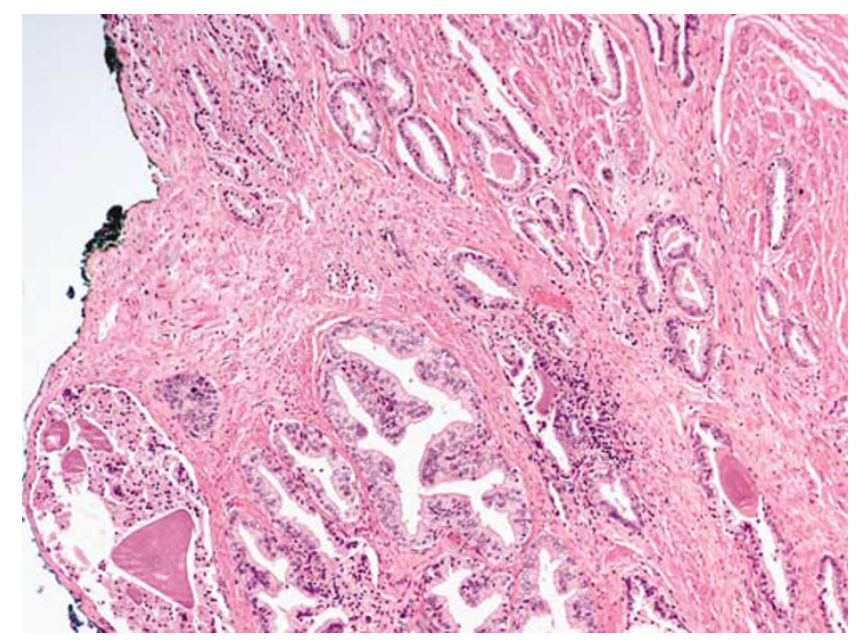

Figure 2 False bladder neck involvement characterized by prostate cancer within the bladder neck section intermixed with benign prostatic glandular tissue.

There was no significant difference $(P=0.51)$ in age between men with bladder neck involvement (median 59 years; range $45-75$ ) and negative bladder neck (median 60 years; range 39-79). Microscopic bladder neck involvement was significantly associated with other poor prognostic signs. Extraprostatic extension was seen in $48 \%$ of men with positive bladder neck vs $25 \%$ of cases with negative bladder neck $(P<0.0001)$; seminal vesicle invasion in $19 \%$ of positive bladder neck vs $5 \%$ of negative bladder neck $(P<0.0001)$; lymph node involvement in $11 \%$ of positive bladder neck vs $1 \%$ of negative bladder neck $(P<0.0001)$; positive surgical margin in $71 \%$ of positive bladder neck involvement $v S$ $22 \%$ of negative bladder neck involvement $(P<0.0001$; Table 1). Eighty-two percent of the positive bladder neck patients had a Gleason score $\geq 7$ vs $55 \%$ of the negative bladder neck $(P<0.0001$ by $\chi^{2}$-test). The tumor volume was extensive in 
Table 1 Operative characteristics of all patients

\begin{tabular}{|c|c|c|c|}
\hline Patient characteristic & $\begin{array}{l}\text { Bladder neck positive } \\
\quad(\mathrm{n}=90)\end{array}$ & $\begin{array}{l}\text { Bladder neck negative } \\
\quad(\mathrm{n}=1755)\end{array}$ & $\mathrm{P}$-value \\
\hline Median age, year (range) & $59(45-75)$ & $60(39-79)$ & $0.51^{\mathrm{a}}$ \\
\hline Median preoperative PSA, ng/ml (range) & $7(1.4-45)$ & $6(0.1-54)$ & $0.0013^{\mathrm{a}}$ \\
\hline Surgical Gleason score (\%) & & & $<0.0001^{\mathrm{b}}$ \\
\hline$\leq 6$ & $16(18)$ & $619(35)$ & \\
\hline 7 & $61(68)$ & $1055(50)$ & \\
\hline$\geq 8$ & $13(14)$ & $78(4)$ & \\
\hline Tumor volume (\%) & & & $<0.0001^{\mathrm{c}}$ \\
\hline Low & $3(3)$ & 329 (19) & \\
\hline Medium & $28(31)$ & $754(43)$ & \\
\hline Extensive & $43(48)$ & $333(19)$ & \\
\hline N/A & $16(18)$ & 339 (19) & \\
\hline Extraprostatic extension (\%) & $43(48)$ & $448(25)$ & $<0.0001^{\mathrm{b}}$ \\
\hline Seminal vesicle invasion (\%) & $17(19)$ & $88(5)$ & $<0.0001^{\mathrm{b}}$ \\
\hline Lymph node involvement (\%) & $10(11)$ & $16(1)$ & $<0.0001^{\mathrm{b}}$ \\
\hline Positive surgical margin (\%) & $64(71)$ & $395(22)$ & $<0.0001^{\mathrm{b}}$ \\
\hline PSA recurrence (\%) & $28(31)$ & $237(13)$ & NA \\
\hline $\begin{array}{l}\text { Median PSA follow-up time (months) in patients without PSA } \\
\text { recurrence (range) }\end{array}$ & $47(1-126)$ & $47(0-139)$ & NA \\
\hline
\end{tabular}

NA, not applicable.

${ }^{\mathrm{a}}$ Unpaired $t$-test.

${ }^{\mathrm{b}} \chi^{2}$-test.

${ }^{\mathrm{C}}$ Fisher's exact test.

Table 2 Surgical margin of resection status in bladder neck positive cases

\begin{tabular}{lcc}
\hline $\begin{array}{l}\text { Microscopic bladder } \\
\text { neck involvement } \\
(\mathrm{n}=90)\end{array}$ & $\begin{array}{c}\text { Positive surgical } \\
\text { margin (\%) at any } \\
\text { location }\end{array}$ & $\begin{array}{c}\text { Positive surgical } \\
\text { margin (\%) at the } \\
\text { bladder neck }\end{array}$ \\
\hline $\begin{array}{l}\text { True bladder neck } \\
\text { involvement } n=63 \\
(70 \%)\end{array}$ & $46 / 63(73 \%)$ & $31 / 63(49 \%)$ \\
$\begin{array}{l}\text { False bladder } \\
\text { involvement } n=27 \\
(30 \%)\end{array}$ & $18 / 27(67 \%)$ & $16 / 27(59 \%)$ \\
& $64 / 90(71 \%)$ & $47 / 90(52 \%)$ \\
\hline
\end{tabular}

almost half $(48 \%)$ of the cases with bladder neck involvement vs $19 \%$ of the cases with negative bladder neck $(P<0.0001$ by Fisher's exact test). The Gleason score and the tumor volume distribution for the two groups of patients are reported in Table 1.

In $48 / 90(53 \%)$ cases with bladder neck involvement, the prostate cancer involved predominantly the anterior zone. True bladder neck involvement was present in $36(75 \%)$ of the 48 cases. Approximately half (47/90) of the cases with bladder neck involvement had also concomitant positive surgical margin at the bladder neck. Positive surgical margin was evident in $17 / 90$ patients (19\%) at sites other than the bladder neck (Table 2).

The median preoperative PSA for the bladder neck positive and bladder neck negative men was $7 \mathrm{ng} / \mathrm{ml}$ (range 1.4-45), and $6 \mathrm{ng} / \mathrm{ml}$ (range 0.1-54), respectively $(P=0.0013)$. Median PSA follow-up time in bladder neck positive and bladder neck negative patients without PSA recurrence was 47 (range 1-126), and 47 months (range 0-139), respectively. PSA recurrence (biochemical failure) occurred in 265 (14\%) patients: 237 (13\%) of the 1755 patients without bladder neck involvement and $28(31 \%)$ of the 90 patients with bladder neck involvement.

Univariate Cox model results indicated that bladder neck involvement was associated with a greater PSA-recurrence risk than negative bladder neck (relative risk 2.71, $P<0.0001$ ): both true bladder neck involvement (relative risk 2.93, $P<0.0001)$ and false bladder neck involvement (relative risk 2.20, $P=0.04$ ) were significantly associated with an increase PSA-recurrence risk. The PSA-recurrence relative risk associated with extraprostatic extension, seminal vesicle invasion, positive surgical margin, lymph node involvement, and PSA was 3.91, 5.71, 4.13, 8.33, and 1.08, respectively $(P<0.0001)$. Gleason score and tumor volume were also associated with a risk of PSA recurrence: Gleason score $\geq 8$ vs Gleason score $\leq 6$, relative risk 21.74, $P<0.0001$; Gleason score $\geq 8$ vs Gleason score 7 , relative risk 5.35, $P<0.0001$; tumor volume extensive vs low, relative risk 2.38, $P<0.001$, tumor volume medium vs low, relative risk 1.99, $P<0.008$.

In the multivariate Cox model controlling for PSA, extraprostatic extension, seminal vesicle invasion, lymph node involvement, Gleason score, tumor volume, and positive surgical margin, the PSArecurrence risk with bladder neck involvement was 


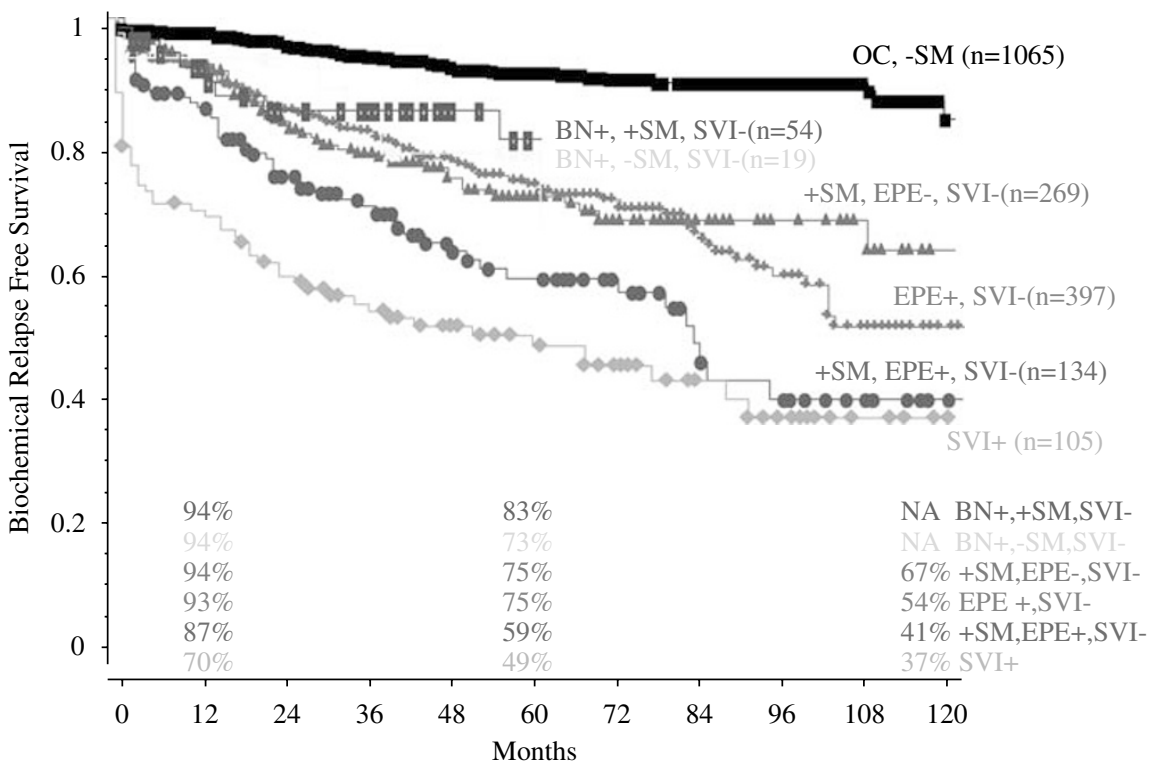

Figure 3 Kaplan-Meier curves for PSA recurrence for different pathological groups of patients. The biochemical relapse-free survival at 12, 60, and 120 months is reported for each group. (1) OC, -SM (organ-confined disease, negative surgical margin; pT2); (2) BN + , + SM, SVI- (bladder neck involvement, positive surgical margin, negative seminal vesicle); (3) BN +, -SM, SVI- (bladder neck involvement, negative surgical margin, negative seminal vesicle); (4) + SM, EPE-, SVI- (positive surgical margin, negative extraprostatic extension, negative seminal vesicle (pT2 + )); (5) EPE +, SVI- (extraprostatic extension, negative seminal vesicle (pT3a)); (6) + SM, EPE +, SVI(positive surgical margin, extraprostatic extension, negative seminal vesicle (pT3a + )); (7) SVI + (seminal vesicle invasion (pT3b)).

not a significant independent prognostic factor. Extraprostatic extension (relative risk 1.89, 95\% CI $1.43-2.48, P<0.001$ ), seminal vesicle invasion (relative risk $1.71,95 \%$ CI $1.20-2.43, P=0.003)$, positive surgical margin (relative risk $2.78,95 \%$ CI 2.16-3.57, $P<0.001$ ), lymph node involvement (relative risk 1.86, 95\% CI 1.09-3.18, $P=0.024$ ), PSA (relative risk 1.06, 95\% CI 1.05-1.08, $P<0.001$ ), and Gleason score (Gleason score $\geq 8$ vs Gleason score $\leq 6$, relative risk 9.09, 95\% CI 5.52-14.92, $P<0.0001$; Gleason score $\geq 8$ vs Gleason score 7, relative risk $3.64,95 \%$ CI $2.60-5.08, P<0.0001$ ) remained as independent predictors of PSA recurrence.

To better categorize the weight of bladder neck involvement relative to other pathological parameters, we compared the PSA-recurrence (biochemical relapse)-free survival in different pathological groups (1, organ-confined disease with negative surgical margin (pT2); 2, bladder neck involvement, positive surgical margin, negative seminal vesicle; 3 , bladder neck involvement, negative surgical margin, negative seminal vesicle; 4 , positive surgical margin, negative extraprostatic extension, negative seminal vesicle (pT2 +); 5, extraprostatic extension, negative seminal vesicle (pT3a); 6, positive surgical margin, extraprostatic extension, negative seminal vesicle (pT3a + ); 7, seminal vesicle invasion (pT3b)) using Cox proportional hazards models. We found that the biochemical relapse-free survival in patients with bladder neck involvement associated with or without positive margin (83 and $73 \%$, respectively) at 60 months was similar to that of pT2 patients with positive surgical margin (75\%) or pT3a patients with negative surgical margin (75\%; Figure 3).

\section{Discussion}

The current clinical and pathological classification of prostate cancer is based on the AJCC TNM staging system. ${ }^{1,2}$ Accurate staging should reflect the natural history of the disease and be able to stratify patients into groups with different prognosis.

The immediate goal of radical prostatectomy therapy for prostate cancer is to achieve biochemical control with undetectable PSA. Biochemical failure precedes clinical failure and is the most commonly detected failure after radical prostatectomy. Adverse pathologic parameters such as extraprostatic extension, seminal vesicle invasion, high Gleason score, and positive surgical margin have been reported to be of value in predicting the likelihood of disease recurrence. ${ }^{16-19}$

The independent prognostic significance of microscopic involvement of the bladder neck by prostate cancer is questionable and controversial as to whether it should be considered just pT3a or pT4a. Bladder neck involvement has been initially considered advanced disease, similar to external sphincter and/or rectal involvement and categorized as pT4 disease. ${ }^{3}$

Despite dramatic stage migration, bladder neck involvement is still a finding in contemporary series of patients undergoing radical prostatectomy, and its 
prevalence ranges from 2.8 to $8.7 \% .^{5-7,13,20,21}$ Similarly, bladder neck involvement was found in $4.9 \%$ radical prostatectomies in this study.

Most of the previous studies ${ }^{4-7,20}$ have found that bladder neck involvement carries a risk of progression similar to extraprostatic extension and lower than seminal vesicle invasion and suggested to consider downstaging bladder neck involvement in the TNM staging system. However, the lack of a consistent pathological definition of bladder neck involvement in different studies complicates the accurate determination of prognostic significance of microscopic bladder neck involvement.

Yossepowitch et $a l^{6}$ compared radical prostatectomy cases with bladder neck involvement (considered equivalent to stage pT4a) versus those with seminal vesicle invasion. In their study, bladder neck invasion was defined as the presence of malignant cells within the thick muscle bundles of the bladder neck sections, without accompanying prostatic glandular tissue. They identified bladder neck involvement in $8.7 \%$ of the 286 cases reviewed and found that bladder neck involvement carries a lower risk of progression than seminal vesicle invasion, although the study was limited by a small number of patients.

Subsequently, Yossepowitch et al $^{7}$ studied a larger cohort of patients (2571 radical prostatectomies) and detected bladder neck invasion (classifies as stage pT4 disease) in $2.8 \%$ of the cases. They also found that the stage pT4 disease comprised a heterogeneous group of tumors with different pathologic features and inconsistent outcome. In a multivariate model, including radical prostatectomy cases, the finding of bladder neck invasion (pT4 disease) did not independently predict PSA recurrence.

Other authors who assessed the predictive importance of bladder neck involvement have considered it as a positive surgical margin at the bladder neck site rather than stage pT4 disease.

Dash et $a l^{5}$ found bladder neck involvement in 5\% of 1123 men with clinically localized prostate cancer treated with radical prostatectomy as monotherapy. In Dash study, bladder neck involvement was defined as margin positivity (extensive involvement) at the base margin of the prostate taken en face. The investigators found a marked difference, in a univariate analysis, in the PSA-recurrence rates for focal (1.52) versus extensive (2.79) involvement of the bladder neck margin, although they admitted that the distinction between focal and extensive was subject to interpretation. However, in the multivariate model, the PSA-recurrence risk with bladder neck involvement was not a significant independent prognostic factor.

In a recent multiethnic, multicenter study of 1722 men treated with radical prostatectomy, Buschemeyer et $a l^{20}$ found that a positive bladder neck margin ( $5 \%$ of patients) was associated with other poor prognostic features, including higher PSA, pathological Gleason score, extraprostatic extension, seminal vesicle invasion, and other positive margins. When concomitant with other positive margins, a positive bladder neck margin was associated with a progression risk similar to that of seminal vesicle invasion (pT3b disease). Although men with an isolated bladder neck margin were reported to have a more favorable pathological profile, they were too few to assess outcome reliably.

The aim of this study was to precisely define microscopic bladder neck involvement and to elucidate the significance of bladder neck involvement. We found that in $70 \%$ of cases, the cancer glands were present in thick smooth muscle bundles characteristic of bladder neck without benign glands. Such bladder neck involvement was considered true bladder neck involvement. In contrast, in $30 \%$ of cases the cancer glands were present together with benign prostate glands in thick smooth muscle bundles. We consider such bladder neck involvement as false. In these cases, it is possible that the surgeon cut into the prostate gland near the bladder neck and that the sampled 'bladder neck' may represent the prostate tissue near the bladder neck.

In this study, bladder neck involvement was associated in the univariate model with a greater PSA-recurrence risk than bladder neck negative cases (relative risk 2.71, $P<0.0001$ ). In addition, both true bladder neck $(P<0.0001)$ and false bladder neck $(P=0.04)$ involvement were associated with an increase PSA-recurrence risk in the univariate model. Bladder neck involvement was not as prognostically unfavorable as extraprostatic extension or seminal vesicle invasion, and the recurrence risk associated with it was intermediate between organ-confined disease (pT2) and extraprostatic extension (pT3). In view of our and other investigators' findings, the AJCC staging system should be revised and the pT4 definition should be modified to include only prostate cancer with gross or radiographic extension into the bladder neck, meanwhile patients with microscopic bladder neck involvement would be better considered equivalent to pT3 disease.

Another important question is whether bladder neck involvement remains as an independent histological prognostic factor like other well-established ones such as Gleason score, extraprostatic extension, and seminal vesicle invasion.

Similar to Buschemeyer et $a l^{20}$ we found that bladder neck involvement was associated with other poor prognostic features such as higher Gleason score and serum PSA, extraprostatic extension, seminal vesicle invasion, positive surgical margin, lymph node involvement, and extensive tumor volume. However, in multivariate model bladder neck involvement (either true or false) was not an independent predictor of PSA recurrence.

Poulos et $a l^{21}$ found bladder neck involvement in $6 \%$ of the 364 patients who underwent surgery for 
prostate cancer at a single institution between 1999 and 2003. Bladder neck involvement was defined as the presence of neoplastic cells within the smooth muscle cells of the coned bladder neck, alike our study. In contrast with our study, bladder neck involvement was an independent predictor of early PSA recurrence. In Poulos et al study, PSA recurrence occurred in $11 \%$ of the 342 patients without bladder neck involvement, whereas $36 \%$ of the 22 patients with bladder neck involvement had PSA recurrence. In our study PSA recurrence occurred in $13.5 \%$ of the 1755 men without bladder neck involvement and in $31 \%$ of the 90 cases with bladder neck involvement. The different results may be related to the small number of cases of Poulos et al study compared to this study as well as the short follow-up (median 12 vs 47 months). ${ }^{21}$

Bladder neck margin evaluation seems important. Blute et al evaluated multiple surgical margins and their impact on PSA failure in 2712 patients and found that bladder neck was the only positive margin site that was a significant predictor of PSA recurrence. $^{11}$

Similarly, Obek et $a 1^{13}$ found positive bladder neck margin to be a significant predictor of PSA recurrence. They detected a positive bladder neck margin in 5\% of the 495 men undergoing radical prostatectomy for clinically localized prostate cancer. Their univariate model demonstrated a significant association between PSA recurrence and Gleason score, extraprostatic extension, seminal vesicle invasion, and positive bladder neck margin. However, their findings are unusual, considering that only Gleason score and positive bladder neck margin were found to be significant in multivariate analysis, meanwhile most studies, including the present one, have found that extraprostatic extension and/or seminal vesicle invasion independently predict PSA recurrence.

In summary, this study demonstrates that bladder neck involvement can be categorized as true and false. Although in univariate model both true and false bladder neck involvement were associated with an increase PSA-recurrence risk, in multivariate analysis, neither true nor false bladder neck involvement was an independent predictor of PSA recurrence. Patients with microscopic bladder neck involvement had a PSA-recurrence risk similar to pT2 and pT3 patients. This study and previous studies strongly argue for staging prostate cancer with microscopic bladder neck invasion as pT2 or pT3 disease.

\section{References}

1 American Joint Committee on Cancer. AJCC Cancer Staging Manual. Lippincott-Raven: New York, 2002.

2 Ohori M, Wheeler TM, Scardino PT. The New American Joint Committee on Cancer and International
Union Against Cancer TNM classification of prostate cancer. Clinicopathologic correlations. Cancer 1994;74:104-114.

3 Fleming ID (ed). AJCC Cancer Staging Manual. Lippincott-Raven: New York, 1997.

4 Billis A, Freitas LL, Magna LA, et al. Prostate cancer with bladder neck involvement: pathologic findings with application of a new practical method for tumor extent evaluation and recurrence-free survival after radical prostatectomy. Int Urol Nephrol 2004;36: 363-368.

5 Dash A, Sanda MG, Yu M, et al. Prostate cancer involving the bladder neck: recurrence-free survival and implications for AJCC staging modification. American Joint Committee on Cancer. Urology 2002;60:276-280.

6 Yossepowitch O, Engelstein D, Konichezky M, et al. Bladder neck involvement at radical prostatectomy: positive margins or advanced T4 disease? Urology 2000;56:448-452.

7 Yossepowitch O, Sircar K, Scardino PT, et al. Bladder neck involvement in pathological stage pT4 radical prostatectomy specimens is not an independent prognostic factor. J Urol 2002;168: 2011-2015.

8 Aydin H, Tsuzuki T, Hernandez D, et al. Positive proximal (bladder neck) margin at radical prostatectomy confers greater risk of biochemical progression. Urology 2004;64:551-555.

9 Srigley JR, Amin MB, Bostwick DG, et al. Updated protocol for the examination of specimens from patients with carcinomas of the prostate gland: a basis for checklists. Cancer Committee. Arch Pathol Lab Med 2000;124:1034-1039.

10 Cheng L, Darson MF, Bergstralh EJ, et al. Correlation of margin status and extraprostatic extension with progression of prostate carcinoma. Cancer 1999;86: 1775-1782.

11 Blute ML, Bostwick DG, Bergstralh EJ, et al. Anatomic site-specific positive margins in organ-confined prostate cancer and its impact on outcome after radical prostatectomy. Urology 1997;50:733-739.

12 Grossfeld GD, Chang JJ, Broering JM, et al. Impact of positive surgical margins on prostate cancer recurrence and the use of secondary cancer treatment: data from the CaPSURE database. J Urol 2000;163: 1171-1177.

13 Obek C, Sadek S, Lai S, et al. Positive surgical margins with radical retropubic prostatectomy: anatomic sitespecific pathologic analysis and impact on prognosis. Urology 1999;54:682-688.

14 Shekarriz B, Tiguert R, Upadhyay J, et al. Impact of location and multifocality of positive surgical margins on disease-free survival following radical prostatectomy: a comparison between African-American and white men. Urology 2000;55:899-903.

15 Renshaw AA, Chang H, D'Amico AV. Estimation of tumor volume in radical prostatectomy specimens in routine clinical practice. Am J Clin Pathol 1997;107: 704-708.

16 Swanson GP, Riggs M, Hermans M. Pathologic findings at radical prostatectomy: risk factors for failure and death. Urol Oncol 2007;25:110-114.

17 Epstein JI, Partin AW, Sauvageot J, et al. Prediction of progression following radical prostatectomy. A multivariate analysis of 721 men with long-term follow-up. Am J Surg Pathol 1996;20:286-292. 
18 Epstein JI, Pound CR, Partin AW, et al. Disease progression following radical prostatectomy in men with Gleason score 7 tumor. J Urol 1998;160:97-100.

19 Roberts WW, Bergstralh EJ, Blute ML, et al. Contemporary identification of patients at high risk of early prostate cancer recurrence after radical retropubic prostatectomy. Urology 2001;57:1033-1037.
20 Buschemeyer III WC, Hamilton RJ, Aronson WJ, et al. Is a positive bladder neck margin truly a T4 lesion in the prostate specific antigen era? Results from the SEARCH Database. J Urol 2008;179:124-129.

21 Poulos CK, Koch MO, Eble JN, et al. Bladder neck invasion is an independent predictor of prostate-specific antigen recurrence. Cancer 2004;101:1563-1568. 